\title{
ON THE SINGULAR STRUCTURE OF THREE-DIMENSIONAL, AREA-MINIMIZING SURFACES ${ }^{1}$ BY
}

FRANK MORGAN

\begin{abstract}
A sufficient condition is given for the union of two three-dimensional planes through the origin in $\mathbf{R}^{n}$ to be area-minimizing. The condition is in terms of the three angles $0 \leqslant \gamma_{1} \leqslant \gamma_{2} \leqslant \gamma_{3}$ which characterize the geometric relationship between the planes. If $\gamma_{3} \leqslant \gamma_{1}+\gamma_{2}$, the union of the planes is area-minimizing.
\end{abstract}

Introduction. Surfaces which are absolutely area-minimizing in the class of locally integral currents in $\mathbf{R}^{n}$ have small and interesting singular sets. $m$-dimensional area-minimizing surfaces in $\mathbf{R}^{m+1}$ are smooth manifolds for $m \leqslant 6$, and for $m \geqslant 7$ their singular sets have Hausdorff dimension at most $m-7$. Larger singular sets occur in higher codimensions. For example, complex analytic varieties, which are automatically area-minimizing, provide real $m$-dimensional surfaces with $(m-2)$ dimensional singular sets. Recent work of Almgren [1] seems to show that the dimension of a singular set never exceeds $m-2$.

Little is known about the structure of the singular set. Only for two-dimensional area-minimizing surfaces is even the first order structure of singularities understood [5]; cf. also [6].

This paper studies the first order structure of singularities in three-dimensional area-minimizing surfaces. At each point in the surface there is a tangent cone (not known to be unique), itself area-minimizing, which records first order behavior. If a tangent cone is a plane with multiplicity one, the surface is locally a smooth manifold. Since the next simplest cone is a sum (i.e., union) of planes, we address the following basic question:

Question. When is the sum of two oriented 3-planes through the origin in $\mathbf{R}^{n}$ area-minimizing?

The geometric relationship between two oriented 3-planes in $\mathbf{R}^{n}$ is characterized by three angles: $0 \leqslant \gamma_{1} \leqslant \gamma_{2} \leqslant \pi / 2, \gamma_{2} \leqslant \gamma_{3} \leqslant \pi-\gamma_{2}$. (See Lemma 1 and remarks.)

We prove a sufficient condition in terms of these three angles.

THEOREM. If $\gamma_{3} \leqslant \gamma_{1}+\gamma_{2}$, the sum of the planes is area-minimizing.

We conjecture that the converse holds as well (except for the trivial case of the same plane with opposite orientations).

Received by the editors October 20, 1981.

1980 Mathematics Subject Classification. Primary 49F22.

Key words and phrases. Area-minimizing, mass-minimizing, singular structure.

'This work was supported in part by NSF Grant MCS-7922051. 
Stability. This theorem provides open sets of pairs of planes whose union is areaminimizing. These examples indicate that singularities in compact area-minimizing surfaces with boundary may be stable-may persist under small variations of the boundaries. Previous work and examples pointed to the instability of singularities.

Other possible tangent cones. This paper does not address the question of when the sum of more than two 3-planes through the origin in $\mathbf{R}^{n}$ is area-minimizing. It is of course necessary that the planes be pairwise area-minimizing. That necessary condition proved to be sufficient for two-dimensional planes, and may be sufficient in higher dimensions as well.

It is an open question whether every three-dimensional area-minimizing cone in $\mathbf{R}^{5}$ is a sum of planes. Harvey and Lawson give an example of a three-dimensional area-minimizing cone in $\mathbf{R}^{6}$ which is not a sum of planes [4, III.3.1]. Bryant gives many examples in $\mathbf{R}^{7}$ [2].

Higher-order behavior at singularities. If a tangent cone at a singularity in a three-dimensional area-minimizing surface is a sum of $k$ distinct planes all occurring with multiplicity one, the surface locally separates into $k$ smooth sheets.

With higher multiplicities, branching may occur. For example, by applying a result of Harvey and Lawson [4, III.3.16] to a minimal surface in $\mathbf{R}^{3}$ with a branch point at the origin, one obtains a three-dimensional area-minimizing surface in $\mathbf{R}^{6}$ with a branch point at the origin. The tangent cone is a 3-plane with multiplicity.

Generalizations to m-dimensional surfaces. The geometric relationship between two oriented $m$-planes in $\mathbf{R}^{n}$ is characterized by $m$ angles: $0 \leqslant \gamma_{1} \leqslant \gamma_{2} \leqslant \cdots \leqslant \gamma_{m-1} \leqslant$ $\pi / 2, \gamma_{m-1} \leqslant \gamma_{m} \leqslant \pi-\gamma_{m-1}$.

CONJECTURE. The nonzero sum of two m-planes is area-minimizing $\Leftrightarrow \gamma_{m} \leqslant \gamma_{0}$ $+\cdots+\gamma_{m-1}$.

REMARK. If $\gamma_{m}=\gamma_{0}+\cdots+\gamma_{m-1}$, the two planes are simultaneously Special Langrangian for some symplectic structure on some $\mathbf{R}^{2 m}$ containing them. Therefore, their sum is automatically area-minimizing.

In general, two $m$ planes in $\mathbf{R}^{2 m}$ are simultaneously Special Lagrangian for some symplectic structure $\Leftrightarrow \sum_{j=1}^{m} \pm \gamma_{j}=0$ for some sequence of \pm signs.

For $m \geqslant 3$, an application of the implicit function theorem to $m$-covectors establishes an open set of pairs of $m$-planes whose sum is area-minimizing.

PROOF OF THE THEOREM. The theorem is proved by exhibiting a 3-covector $\phi$ which attains its maximum $M$ on the two given planes (in competition with all other unit simple 3-vectors). Hence the sum of the planes is area-minimizing as follows.

Let $S$ be the portion of the sum of the two planes inside a large ball about 0 , and let $T$ be any other surface with the same boundary. Then

$$
\int_{S} \phi=(\operatorname{area} S) M, \quad \int_{T} \phi \leqslant(\operatorname{area} T) M .
$$

But since $d \phi=0, \int_{S} \phi=\int_{T} \phi$ and hence area $S \leqslant$ area $T$. (We have informally written "area" for the mass of the integral current.)

The theorem. We begin with a canonical form for a pair $\xi_{+}, \xi_{-}$of $m$-planes through the origin in $\mathbf{R}^{2 m}$ which exhibits $m$ angles that characterize their geometric relationship. 
1. Lemma. Let $\xi_{+}, \xi_{-}$be unit simple m-vectors in $\Lambda_{m} \mathbf{R}^{2 m}$. Then there is an orthonormal basis $e_{1}, \ldots, e_{m}, i e_{1}, \ldots, i e_{m}$ for $\mathbf{R}^{2 m} \cong \mathbf{C}^{m}$ such that

$$
\xi_{ \pm}=\exp \left( \pm i \alpha_{1}\right) e_{1} \wedge \cdots \wedge \exp \left( \pm i \alpha_{m}\right) e_{m},
$$

with $0 \leqslant \alpha_{1} \leqslant \cdots \leqslant \alpha_{m-1} \leqslant \pi / 4, \alpha_{m-1} \leqslant \alpha_{m} \leqslant \pi / 2-\alpha_{m-1}$.

REMARK 1. The $\alpha_{j}$ are unique. Another sum of planes $\xi_{+}^{\prime}+\xi_{-}^{\prime}$ is related to $\xi_{+}+\xi_{-}$ by an isometry of $\mathbf{R}^{2 m}$ if and only if $\alpha_{j}^{\prime}=\alpha_{j}$.

REMARK 2. The proof is the same as the proof for 2-planes in [5, Lemma 1], which gives a geometric interpretation to the characterizing angles $\gamma_{j}=2 \alpha_{j}$ (called $\alpha$ and $\beta$ in [5]) between the planes. In an alternative proof [4, II.7.5], Harvey and Lawson let $\pi$ denote projection of $\xi_{+}$into $\xi_{-}$and consider the bilinear form on $\xi_{+}$defined by $B(u, v)=\pi(u) \cdot \pi(v)$. The eigenvalues of $B$ turn out to be the cosines of the angles $\gamma_{j}$.

Before proceeding to the theorem, we give a trigonometric lemma we will need later.

2. LEMMA. Suppose

(a) $0<\alpha_{i}<\alpha_{j}+\alpha_{k}<\pi / 2$ for $\{i, j, k\}=\{1,2,3\}$.

Then

$$
2 \cos \alpha_{1} \cos \alpha_{2} \cos \alpha_{3}>\cos ^{2} \alpha_{1}+\cos ^{2} \alpha_{2}+\cos ^{2} \alpha_{3}-1>0 .
$$

If

$$
\mu_{i}=\cot \alpha_{j} \cot \alpha_{k}\left(\frac{2 \cos ^{2} \alpha_{i}}{\cos ^{2} \alpha_{1}+\cos ^{2} \alpha_{2}+\cos ^{2} \alpha_{3}-1}-1\right)
$$

then

$$
\mu_{i}+\mu_{j}>0, \quad\left|\mu_{i}\right|<1,
$$

and

$$
\frac{1-\mu_{i}}{\mu_{j}+\mu_{k}}=\frac{\tan \alpha_{i}}{\tan \left(\alpha_{j}+\alpha_{k}\right)}<1 .
$$

Proof. All statements apply for $\{i, j, k\}=\{1,2,3\}$. Abbreviate $c_{i}=\cos \alpha_{i}$, $s_{i}=\sin \alpha_{i}, t_{i}=\tan \alpha_{i}, C=c_{1}^{2}+c_{2}^{2}+c_{3}^{2}-1$. By (a), two of the $\alpha_{i}$ are less than $\pi / 4$ and hence $C>0$. Now

$$
\begin{aligned}
0 & <\left|\alpha_{1}-\alpha_{2}\right|<\alpha_{3}<\alpha_{1}+\alpha_{2}<\pi / 2 \\
& \Rightarrow \cos \left(\alpha_{1}-\alpha_{2}\right)>\cos \alpha_{3}>\cos \left(\alpha_{1}+\alpha_{2}\right) \\
& \Rightarrow c_{1} c_{2}+s_{1} s_{2}>c_{3}>c_{1} c_{2}-s_{1} s_{2} \\
& \Rightarrow\left|c_{3}-c_{1} c_{2}\right|<s_{1} s_{2} .
\end{aligned}
$$

Squaring both sides yields

$$
c_{3}^{2}-2 c_{1} c_{2} c_{3}+c_{1}^{2} c_{2}^{2}<\left(1-c_{1}^{2}\right)\left(1-c_{2}^{2}\right) .
$$

Therefore

$$
2 c_{1} c_{2} c_{3}>c_{1}^{2}+c_{2}^{2}+c_{3}^{2}-1
$$

proving (b). 
Now using (c) we compute that

$$
\begin{aligned}
& t_{i} C\left(-t_{1} t_{2} t_{3} \mu_{i}+t_{k} \mu_{j}+t_{j} \mu_{k}-t_{i}\right) \\
& \quad=-t_{i}^{2}\left(2 \cos ^{2} \alpha_{i}-C\right)+\left(2 \cos ^{2} \alpha_{j}-C\right)+\left(2 \cos ^{2} \alpha_{k}-C\right)-t_{i}^{2} C \\
& \quad=0 .
\end{aligned}
$$

Therefore

$$
-t_{1} t_{2} t_{3} \mu_{i}+t_{k} \mu_{j}+t_{j} \mu_{k}=t_{i} \text {. }
$$

Adding equations $\left(\mathrm{f}_{i}\right)$ and $\left(\mathrm{f}_{j}\right)$ and dividing by $1-t_{i} t_{j}$ yields

$$
\left(1-\mu_{k}\right) \tan \left(\alpha_{i}+\alpha_{j}\right)=\left(\mu_{i}+\mu_{j}\right) \tan \alpha_{k} .
$$

Now for convenience we assume $\alpha_{1} \leqslant \alpha_{2} \leqslant \alpha_{3}$. It follows immediately from (c) that $\mu_{1} \geqslant \mu_{2} \geqslant \mu_{3}, \mu_{1}$ and $\mu_{2}$ are positive, and then by $\left(g_{3}\right)$ that

$$
\mu_{1}+\mu_{2}+\mu_{3}>1 \text {. }
$$

Also, $\mu_{2}+\mu_{3}>0$, because if $\mu_{2}+\mu_{3} \leqslant 0$, by $\left(\mathrm{g}_{1}\right) \mu_{1}+\mu_{2}+\mu_{3} \leqslant 1$, a contradiction of (h). By $\left(\mathrm{g}_{1}\right)$ and $\left(\mathrm{g}_{2}\right), \mu_{1}<1$ and $\mu_{2}<1$. Since $\mu_{3} \leqslant \mu_{2}$ and $\mu_{2}+\mu_{3}>0$, $\left|\mu_{3}\right|<1$, and (d) is proved. Now (g) implies the equality in (e). The inequality in (e) follows from (a).

3. TheOREM. Let $\xi_{+}$, $\xi_{-}$be unit simple 3-vectors in $\mathbf{R}^{n}$, and let $S_{1}, S_{2}$ be the locally integral currents associated with those planes. Let $0 \leqslant \alpha_{1} \leqslant \alpha_{2} \leqslant \alpha_{3}$ be the associated angles as in Lemma 1. Then if $\alpha_{3} \leqslant \alpha_{1}+\alpha_{2}, S_{1}+S_{2}$ is area-minimizing.

Proof. We can assume $n=6$. Also, it suffices to consider $\alpha_{i}>0, \alpha_{3}<\alpha_{1}+\alpha_{2}$, since other cases are limits of such. By Lemma 1, we can assume

$$
\xi_{ \pm}=\exp \left( \pm i \alpha_{1}\right) e_{1} \wedge \exp \left( \pm i \alpha_{2}\right) e_{2} \wedge \exp \left( \pm i \alpha_{3}\right) e_{3} .
$$

Now we are ready to define a 3-covector $\phi$ which will attain its maximum on $\xi_{+}$and $\xi$.

Define numbers $\mu_{1}, \mu_{2}, \mu_{3}$ as in 2(c). Let $e_{1}^{*}, e_{2}^{*}, e_{3}^{*},\left(i e_{1}\right)^{*},\left(i e_{2}\right)^{*},\left(i e_{3}\right)^{*}$ be the dual basis for $\Lambda^{2} \mathbf{R}^{6}$, and put

$$
\begin{aligned}
\phi= & e_{1}^{*} \wedge e_{2}^{*} \wedge e_{3}^{*}+\mu_{1} e_{1}^{*} \wedge\left(i e_{2}\right)^{*} \wedge\left(i e_{3}\right)^{*} \\
& +\mu_{2}\left(i e_{1}\right)^{*} \wedge e_{2}^{*} \wedge\left(i e_{3}\right)^{*}+\mu_{3}\left(i e_{1}\right)^{*} \wedge\left(i e_{2}\right)^{*} \wedge e_{3}^{*} .
\end{aligned}
$$

The rest of the proof will show that

$$
\phi\left(\xi_{+}\right)=\phi\left(\xi_{-}\right)=\max \{\phi(\xi): \xi \text { unit, simple 3-vector }\},
$$

from which it follows easily that $S_{1}+S_{2}$ is area-minimizing.

4. LEMMA. Consider $\mathbf{R}^{2 m} \cong \mathbf{C}^{m}$ with orthonormal basis $e_{1}, \ldots, e_{m}, i e_{1}, \ldots, i e_{m}$. Let $\phi \in \Lambda^{m}\left(\mathbf{R}^{2 m}\right)$ satisfy

$$
\phi\left\llcorner e_{j} \wedge i e_{j}=0 \quad(1 \leqslant j \leqslant m) .\right.
$$

Then on the space of unit, simple m-vectors, $\phi$ attains its maximum on an m-vector of the form

$$
\exp \left(i \zeta_{1}\right) e_{1} \wedge \exp \left(i \zeta_{2}\right) e_{2} \wedge \cdots \wedge \exp \left(i \zeta_{m}\right) e_{m}
$$


Proof. Let $\xi$ be a unit, simple $m$-vector on which $\phi$ attains its maximum. Choose a unit vector $u$ in the plane $\xi$ to maximize $u \cdot e_{1}$. Choose a unit vector $v$ in $\xi$ with $v \cdot u=0$ to maximize $v \cdot\left(i e_{1}\right)$. For any vector $x$ denote by $x^{*}$ the dual covector. Let $\eta=\xi \mathrm{L}\left(u^{*} \wedge v^{*}\right)$, so that $\xi=u \wedge v \wedge \eta$. By choice of $u$ and $v$,

$$
\eta \mathrm{L} e_{1}^{*}=\eta \mathrm{L} i e_{1}^{*}=0 \text {. }
$$

For any $x \in \mathbf{R}^{2 m}$, let $x^{\prime}=\left(x \cdot e_{1}\right) e_{1}+\left(x \cdot i e_{1}\right) i e_{1}, x^{\prime \prime}=x-x^{\prime}$. Let

$$
\begin{aligned}
u^{\prime}=\left(\cos \kappa_{0}\right) \hat{u}^{\prime}, & v^{\prime}=\left(\cos \lambda_{0}\right) \hat{v}^{\prime}, \\
u^{\prime \prime}=\left(\sin \kappa_{0}\right) \hat{u}^{\prime \prime}, & v^{\prime \prime}=\left(\sin \lambda_{0}\right) \hat{v}^{\prime \prime},
\end{aligned}
$$

and consider variations in $u$ and $v$ of the form

$$
u(\kappa)=(\cos \kappa) \hat{u}^{\prime}+(\sin \kappa) \hat{\mu}^{\prime \prime}, \quad v(\lambda)=(\cos \lambda) \hat{v}^{\prime}+(\sin \lambda) \hat{v}^{\prime \prime} .
$$

To compute $\phi(u(\kappa) \wedge v(\lambda) \wedge \eta)$, we note that by (1), $\phi\left(\hat{u}^{\prime} \wedge \hat{v}^{\prime} \wedge \eta\right)=0$; also by (1), since every term in the expansion of $\hat{u}^{\prime \prime} \wedge \hat{v}^{\prime \prime} \wedge \eta$ has some $e_{j} \wedge i e_{j}$ as a factor, $\phi\left(\hat{u}^{\prime \prime} \wedge \hat{v}^{\prime \prime} \wedge \eta\right)=0$. Therefore

$$
\begin{aligned}
\phi(u(\kappa) \wedge & v(\lambda) \wedge \eta) \\
& =\phi\left((\cos \kappa) \hat{u}^{\prime} \wedge(\sin \lambda) \hat{v}^{\prime \prime} \wedge \eta\right)+\phi\left((\sin \kappa) \hat{u}^{\prime \prime} \wedge(\cos \lambda) \hat{v}^{\prime} \wedge \eta\right) \\
& =a \cos \kappa \sin \lambda+b \sin \kappa \cos \lambda,
\end{aligned}
$$

for some $a, b$ independent of $\kappa, \lambda$.

Since this function of $\kappa$ and $\lambda$ has a maximum at $\kappa_{0}, \lambda_{0}$, its partial derivatives vanish there:

$$
\begin{aligned}
& -a \sin \kappa_{0} \sin \lambda_{0}+b \cos \kappa_{0} \cos \lambda_{0}=0, \\
& -b \sin \kappa_{0} \sin \lambda_{0}+a \cos \kappa_{0} \cos \lambda_{0}=0 .
\end{aligned}
$$

Subtracting $b$ times the second from $a$ times the first, and $a$ times the second from $b$ times the first yields

$$
\left(b^{2}-a^{2}\right) \sin \kappa_{0} \sin \lambda_{0}=\left(b^{2}-a^{2}\right) \cos \kappa_{0} \cos \lambda_{0}=0 .
$$

If $a= \pm b$,

$$
a \cos \kappa \sin \lambda+b \sin \kappa \cos \lambda=b \sin (\kappa \pm \lambda),
$$

and we can assume $\kappa_{0}=0, \lambda_{0}= \pm \pi / 2$. Otherwise, $\sin \kappa_{0}=\cos \lambda_{0}=0$ or $\cos \kappa_{0}=$ $\sin \lambda_{0}=0$. In any case, $\xi$ is of the form

$$
\xi=\exp \left(i \zeta_{1}\right) e_{1} \wedge \xi^{\prime}
$$

with $\xi^{\prime}$ a simple unit $(m-1)$-vector in

$$
\Lambda_{m-1}\left(\operatorname{span}\left\{e_{2}, i e_{2}, \ldots, e_{m}, i e_{m}\right\}\right) \cong \Lambda_{m-1} \mathbf{R}^{2(m-1)} .
$$

Now $\phi \operatorname{Lexp}\left(i \zeta_{1}\right) e_{1} \in \Lambda^{m-1}\left(\mathbf{R}^{2(m-1)}\right)$ and $\phi \operatorname{Lexp}\left(i \zeta_{1}\right) e_{1}$ attains its maximum on $\xi^{\prime}$. Hence by induction we can assume

$$
\xi^{\prime}=\left(\exp i \zeta_{2}\right) e_{2} \wedge \cdots \wedge\left(\exp i \zeta_{m}\right) e_{m}
$$

and the lemma is proved.

Now to establish 3(2), it suffices to show that

$$
\phi\left(\exp \left(i \zeta_{1}\right) e_{1} \wedge \exp \left(i \zeta_{2}\right) e_{2} \wedge \exp \left(i \zeta_{3}\right) e_{3}\right)
$$

attains its maximum at $\zeta_{i}= \pm \alpha_{i}$. 


\section{Lemma. Define}

$$
f\left(\zeta_{1}, \zeta_{2}, \zeta_{3}\right)=\phi\left(\exp \left(i \zeta_{1}\right) e_{1} \wedge \exp \left(i \zeta_{2}\right) e_{2} \wedge \exp \left(i \zeta_{3}\right) e_{3}\right)
$$

Then the 48 critical points of $f(\bmod 2 \pi)$ are

$$
\begin{gathered}
\left(\frac{\pi}{2} \pm \frac{\pi}{2}, \pm \frac{\pi}{2}, \pm \frac{\pi}{2}\right),\left( \pm \frac{\pi}{2}, \frac{\pi}{2} \pm \frac{\pi}{2}, \pm \frac{\pi}{2}\right),\left( \pm \frac{\pi}{2}, \pm \frac{\pi}{2}, \frac{\pi}{2} \pm \frac{\pi}{2}\right), \\
\left(\frac{\pi}{2} \pm \frac{\pi}{2}, \frac{\pi}{2} \pm \frac{\pi}{2}, \frac{\pi}{2} \pm \frac{\pi}{2}\right), \pm\left(\alpha_{1}+\frac{\pi}{2} \pm \frac{\pi}{2}, \alpha_{2}+\frac{\pi}{2} \pm \frac{\pi}{2}, \alpha_{3}+\frac{\pi}{2} \pm \frac{\pi}{2}\right) .
\end{gathered}
$$

$f$ attains its maximum at $\pm\left(\alpha_{1}, \alpha_{2}, \alpha_{3}\right)$.

Proof. All statements apply for $\{i, j, k\}=\{1,2,3\}$. We compute that

$$
\begin{aligned}
f\left(\zeta_{1}, \zeta_{2}, \zeta_{3}\right)= & \cos \zeta_{1} \cos \zeta_{2} \cos \zeta_{3}+\mu_{1} \cos \zeta_{1} \sin \zeta_{2} \sin \zeta_{3} \\
& +\mu_{2} \sin \zeta_{1} \cos \zeta_{2} \sin \zeta_{3}+\mu_{3} \sin \zeta_{1} \sin \zeta_{2} \cos \zeta_{3} .
\end{aligned}
$$

Let $\left(\beta_{1}, \beta_{2}, \beta_{3}\right)$ be a critical point, and abbreviate $c_{i}=\cos \beta_{i}, s_{i}=\sin \beta_{i}$. The partial derivative of $f$ with respect to $\zeta_{i}$ must vanish at $\left(\beta_{1}, \beta_{2}, \beta_{3}\right)$ :

$$
-s_{i} c_{j} c_{k}-\mu_{i} s_{i} s_{j} s_{k}+\mu_{j} c_{i} c_{j} s_{k}+\mu_{k} c_{i} s_{j} c_{k}=0 \text {. }
$$

Adding equations $\left(\mathrm{a}_{j}\right)$ and $\left(\mathrm{a}_{k}\right)$ yields

$$
-c_{i} \sin \left(\beta_{j}+\beta_{k}\right)\left(1-\mu_{i}\right)+\left(\mu_{j}+\mu_{k}\right) s_{i} \cos \left(\beta_{j}+\beta_{k}\right)=0 .
$$

Case I. Some $\beta_{i} \in \frac{\pi}{2} \mathbf{Z}$ or some $\beta_{j}+\beta_{j} \in \frac{\pi}{2} \mathbf{Z}$.

If $c_{i}=0$, since $\mu_{j}+\mu_{k}>0$, by $2(\mathrm{~d}),\left(\mathrm{b}_{i}\right)$ implies that $c_{j} c_{k}-s_{j} s_{k}=0$. By $\left(\mathrm{a}_{i}\right)$, $c_{j} c_{k}+\mu_{i} s_{j} s_{k}=0$. Hence, $\left(1+\mu_{i}\right) s_{j} s_{k}=0$. Since $\left|\mu_{i}\right|<1$, either $s_{j}=0$ and $c_{k}=0$ or $s_{k}=0$ and $c_{j}=0$.

If $s_{i}=0,\left(\mathrm{~b}_{i}\right)$ implies that $c_{j} s_{k}+s_{j} c_{k}=0$. By $\left(\mathrm{a}_{k}\right),-c_{j} s_{k}+\mu_{i} s_{j} c_{k}=0$. Now either $s_{j}=0$ and $s_{k}=0$ or $c_{j}=0$ and $c_{k}=0$.

If $\beta_{i} \neq \frac{\pi}{2} \mathbf{Z}$, by $\left(b_{i}\right), \beta_{j}+\beta_{k} \notin \frac{\pi}{2} \mathbf{Z}$.

Therefore, Case I yields the following 32 critical points:

$$
\left(\frac{\pi}{2} \pm \frac{\pi}{2}, \pm \frac{\pi}{2}, \pm \frac{\pi}{2}\right),\left( \pm \frac{\pi}{2}, \frac{\pi}{2} \pm \frac{\pi}{2}, \pm \frac{\pi}{2}\right),\left( \pm \frac{\pi}{2}, \pm \frac{\pi}{2}, \frac{\pi}{2} \pm \frac{\pi}{2}\right),\left(\frac{\pi}{2} \pm \frac{\pi}{2}, \frac{\pi}{2} \pm \frac{\pi}{2}, \frac{\pi}{2} \pm \frac{\pi}{2}\right) \text {. }
$$

Case II. No $\beta_{i}$ or $\beta_{j}+\beta_{k} \in \frac{\pi}{2}$ Z. By (b) and 2(e),

$$
\frac{\tan \beta_{i}}{\tan \left(\beta_{j}+\beta_{k}\right)}=\frac{1-\mu_{i}}{\mu_{j}+\mu_{k}}=\frac{\tan \alpha_{i}}{\tan \left(\alpha_{j}+\alpha_{k}\right)} \equiv p_{i},
$$

and $0<p_{i}<1$. But if $\left(\tan \zeta_{i}\right) / \tan \left(\zeta_{j}+\zeta_{k}\right)=p_{i}, t_{i}=\tan \zeta_{i}$,

$$
-t_{i}+p_{i} t_{j}+p_{i} t_{k}+t_{1} t_{2} t_{3}=0 \text {. }
$$

Multiplying $\left(\mathrm{c}_{i}\right)$ by $-\left(1+p_{j}\right),\left(\mathrm{c}_{j}\right)$ by $\left(1+p_{i}\right),\left(\mathrm{c}_{k}\right)$ by $\left(p_{j}-p_{i}\right)$ and adding yields

$$
\left[1+2 p_{j}+p_{i} p_{j}+p_{j} p_{k}-p_{i} p_{k}\right] t_{i}=\left[p_{i} p_{j}+2 p_{i}+1-p_{j} p_{k}+p_{i} p_{k}\right] t_{j} \text {. }
$$

Since $0<p_{i}<1$, both coefficients are positive. Since these equations hold for $t_{i}=\tan \beta_{i}$ and for $t_{i}=\tan \alpha_{i}$, we conclude that for some $k \in \mathbf{R}, \tan \beta_{i}=k \tan \alpha_{i}$. Then $\left(\mathrm{c}_{1}\right)$ implies $k \in\{-1,0,1\}$. Since $\beta_{i} \notin \frac{\pi}{2} \mathbf{Z}, k= \pm 1$. The 16 critical points of Case II are $\pm\left(\alpha_{1}+\frac{\pi}{2} \pm \frac{\pi}{2}, \alpha_{2}+\frac{\pi}{2} \pm \frac{\pi}{2}, \alpha_{3}+\frac{\pi}{2} \pm \frac{\pi}{2}\right)$.

Finally, we show that $f$ attains its maximum at $\pm\left(\alpha_{1}, \alpha_{2}, \alpha_{3}\right)$. The values of $f$ at its critical points are $\pm \mu_{1}, \pm \mu_{2}, \pm \mu_{3}, \pm 1, \pm f\left(\alpha_{1}, \alpha_{2}, \alpha_{3}\right)$. Since $\left|\mu_{i}\right|<1$ and 
$f\left(\alpha_{1}, \alpha_{2}, \alpha_{3}\right)=f\left(-\alpha_{1},-\alpha_{2},-\alpha_{3}\right)$, it suffices to show that $f\left(\alpha_{1}, \alpha_{2}, \alpha_{3}\right) \geqslant 1$. But, abbreviating $t_{;}=\tan \alpha_{i}$,

$$
\begin{aligned}
f\left(\alpha_{1}, \alpha_{2}, \alpha_{3}\right) & =\cos \alpha_{1} \cos \alpha_{2} \cos \alpha_{3}\left(1+t_{2} t_{3} \mu_{1}+t_{1} t_{3} \mu_{2}+t_{1} t_{2} \mu_{3}\right) \\
& =\cos \alpha_{1} \cos \alpha_{2} \cos \alpha_{3}\left(1+\frac{2 \cos ^{2} \alpha_{1}+2 \cos ^{2} \alpha_{2}+2 \cos ^{2} \alpha_{3}}{\cos ^{2} \alpha_{1}+\cos ^{2} \alpha_{2}+\cos ^{2} \alpha_{3}-1}-3\right)
\end{aligned}
$$

by the definition of the $\mu_{i}$

$$
=\frac{2 \cos \alpha_{1} \cos \alpha_{2} \cos \alpha_{3}}{\cos ^{2} \alpha_{1}+\cos ^{2} \alpha_{2}+\cos ^{2} \alpha_{3}-1}>1 \text { by } 2(\mathrm{~b}) \text {. }
$$

Completion of Proof. By Lemmas 4 and 5, $\phi$ attains its maximum on $\xi_{+}$and on $\xi_{\text {. }}$. Hence, by a well-known argument (see Introduction) $S_{1}+S_{2}$ is area-minimizing.

\section{REFERENCES}

1. F. J. Almgren, Jr., Multiple valued functions minimizing Dirichlet's integral and the regularity of mass minimizing integral currents (in preparation).

2. R. Bryant, Submanifolds and special structures on the octonions, J. Differential Geometry 17 (1982), 185-232.

3. Herbert Federer, Geometric measure theory, Springer-Verlag, New York, 1969.

4. Reese Harvey and H. Blaine Lawson, Jr., Calibrated geometries, Acta Math. (to appear).

5. Frank Morgan, On the singular structure of two-dimensional area-minimizing surfaces in $\mathbf{R}^{n}$, Math. Ann. (to appear).

6. Jean E. Taylor, The structure of singularities in soap-bubble-like and soap-film-like minimal surfaces, Ann. of Math. (2) 103 (1976), 489-539.

Department of Mathematics, Massachusetts Institute of Technology, Cambridge, MasSACHUSETTS 02139 